\title{
A Trust Vector Approach to Service-Oriented Applications
}

\author{
Lei Li \\ Department of Computing \\ Macquarie University \\ Sydney, NSW 2109, Australia \\ leili@ics.mq.edu.au
}

\author{
Yan Wang \\ Department of Computing \\ Macquarie University \\ Sydney, NSW 2109, Australia \\ yanwang@ics.mq.edu.au
}

\begin{abstract}
Trust is a critical issue in e-commerce and e-service environments. In some applications (such as eBay), the trust management mechanisms have been introduced to provide valuable information to buyers prior to placing orders and making payments. Meanwhile, the trust issue is also actively studied in the research community. However, most studies and applications focus on approaches that result in a single trust value to represent the trust level of sellers or service providers. Such a simple trust evaluation method may not be able to depict the trust history exactly and may leave misleading information to service customers. In this paper, we present a novel approach of trust vector consisting of three values to reflect the trust level with more indications.
\end{abstract}

\section{Introduction}

In e-commerce or e-service environments, the reputation-based trust status of a seller or a service provider is a very important concern from the view point of a buyer or a service customer. When there are a few sellers or service providers providing the same product or service, the buyer or service customer would like to order from the seller or service provider with the best transaction reputation. This is particularly important when the buyer or service customer has to select from unknown sellers or service providers.

In general, in a trust management mechanism enabled system, buyers or service customers can provide feedback and ratings after transactions. Then the trust management system can calculate the trust value based on collected ratings reflecting the quality of recent transactions. The trust value can be provided to buyers or service customers, by publishing it on web or responding to their requests.

Peer-to-Peer (P2P) networks can be used for information-sharing systems [2], where the trust issue is also actively studied (e.g. [12]). In a P2P system, it is quite natural for a client peer to doubt if a serving peer can provide the complete file prior to any download action, which may be quite time-consuming and network bandwidth-consuming. Different from some trust management systems in e-commerce environments, in the $\mathrm{P} 2 \mathrm{P}$ trust system, a requesting peer needs to enquire the trust data of a serving peer (target peer) from other peers which may have transacted with the serving peer $[4,6,13]$. The computation of the trust status of the serving peer from the collected trust ratings is then performed by the requesting peer, not a central management server, because of the decentralized architecture of P2P system.

In existing trust management studies, the final trust level is computed to reflect the general or global trust status of every service provider. For example, if the final trust level value is in the range of $[0,1], 0.95$ indicates a very good trust level for a service provider. Usually the final trust value is computed by taking the service trust ratings in a recent period into account, i.e. assigning higher weights to trust values of recent transactions or services as the latest transaction rating is the most important in trust evaluation [11][15].

Such a final trust level value can reflect the service reputation accumulated in a certain period. However, a single final trust level value cannot reflect the real trust status very well. For example, the single-value approach cannot reflect (i) the service trust trend of changes and (ii) the service performance consistency level. Service trust trend indicates that the service trust will become better or worse in forthcoming transactions. This is an important indication to tell buyers or service customers to what extent a seller or a service provider is trustworthy for new transactions or services. Service performance consistency level can indicate whether the service quality is being maintained at the level reflected by the final trust value, which makes sense no matter whether the trust value is low or high. For example, if the trust level of a service provider is good and its trust level is "consistent", it indicates that the service provider has maintained the good level for a certain period. 
Thus a good trust management system requires more comprehensive trust evaluation approaches providing more (precise) trust information that indicates not only the global trust level, but also the trust prediction relevant to forthcoming transactions. To serve for this purpose, in this paper, we propose a service trust vector consisting of a set of values, such as final trust level, service trust trend and service performance consistency level, which is applicable to e-commerce or e-service environments. We will also conduct empirical studies to study the properties of our proposed approaches.

This paper is organized as follows. In Section 2, we review the trust management approaches of eBay and some existing studies. Section 3 discusses service trust trend and service performance consistency level evaluations in our service trust vector. Some empirical studies are presented in Section 4 for further illustrating the properties of our model. Finally Section 5 concludes our work.

\section{Background}

\subsection{Trust Management at eBay}

eBay [1] is a typical Customer-to-Customer (C2C) web site, whose trust management mechanism is one of the earliest systems in applications.

At eBay, after each transaction, the buyer can give feedback to the system according to the service quality of the seller. The feedback (or rating) is stored by eBay (a centralized management architecture), which can be "positive", "neutral" or "negative". eBay calculates the feedback score $S=P-N$, where $P$ is the number of positive feedback left by members (customers) and $N$ is the number of negative feedback from members. Then $S$ value can be displayed on the web pages. In addition, $R=\frac{P-N}{P+N}$ (e.g., $R=99.1 \%$ ) is called positive feedback rate, based on which a seller can be awarded as a "Power Seller" if $R \geq 98 \%$ (98\% is the threshold).

eBay also improves its trust service and provides rating data in 12 months listed in a table, which is divided by recent 1 month, 6 months and 12 months. Thus, eBay provides some simple mechanisms of trust management and trust calculation and leaves some raw data to buyers for self-calculation. Obviously, it leaves much room for improvement.

\subsection{Trust Management in Other Environments}

In [15], a trust evaluation approach is proposed for ecommerce applications which is based on the trust values of transactions in a recent period, rather than all of them. In this method, recent ratings are more important in the trust evaluation. In [8], fuzzy logic is applied to trust evaluation, which divides sellers or service providers into multiple classes of reputation ranks (e.g. a 5-star seller, or a 4-star seller).

Trust issue is also actively pursued in Peer-to-Peer (P2P) information-sharing network (e.g. [2]). P2P trust evaluation relies on a polling algorithm (e.g. [3]), a binary rating system for calculating the global trust value of a given peer [4][13], or a voting reputation system (e.g. [6]) that calculates the final trust value combining the values returned by responding peers and the requesting peer's experience with the given peer.

As pointed in [14], binary ratings work pretty well for file-sharing systems where a file is either the definitive correct version or wrong, but cannot accurately model richer services such as web services or e-commerce, where a Boolean may not adequately represent a client peer's experience of the quality of service (QoS) with other serving peers, such as the quality of products the serving peer sends and the expected delivery time [14]. In most later studies on trust evaluation (e.g. [11], [12], [14]), a numeral rating system is adopted, where, for example, the rating is a value in the range of $[0,1]$. Such a rating system is more suitable for complex applications, such as e-commerce or serviceoriented applications.

In the literature, trust issue also receives much attention in service-oriented computing research. In [5], Lin et al propose a method of reputation-based trust evaluation in service-oriented environments based on a proposed architecture consisting of distributed trust management brokers. In [9], Vu et al present a model to evaluate and rank the trust and reputation of QoS-based services. In [10], an event-driven and rule-based trust management for serviceoriented application is proposed, where a formula based approach is adopted for incremental trust computation. Moreover, the approach is adaptable to applications by incorporating rule management. Then, the computed result can be taken as a global trust value reflecting the accumulated trust level, which is not particularly relevant to forthcoming transactions.

\subsection{Some Issues in Trust Evaluation}

However, in most existing studies, a single-value service trust evaluation method is adopted, which is simple but cannot reflect the trust status precisely. In general, the $f-$ nal trust level (FTL) value is provided as an indication for the global trust level for a seller or service provider, which may be presumably taken as a prediction of trustworthiness for forthcoming transactions. Namely, if a seller or service provider has a very good $F T L$ value, a forthcoming transaction will be very trustworthy; otherwise, it is not trustworthy. Obviously, this may be misleading. More information should be provided to reflect the global trust more precisely, which can be valuable to predict the trust level of a new transaction well. This information includes some aspects as 
follows.

1. The service trust trend (STT) value should be computed which is useful to predict the future trust level of service quality.

$S T T$ value shows a general trend of changes in the service quality in the near future, which is important when we choose a seller or service provider with serious caution. Namely, given a set of sellers or service providers with the same $F T L$ value, the one which is becoming better is more desirable to a buyer or service customer. Obviously, with a single $F T L$ value only, it is impossible to illustrate this property.

2. The service performance consistency level (SPCL) value should be calculated to indicate whether the real service level is consistent in a certain period.

Given a set of sellers or service providers with the same FTL and STT values, the one which has higher consistency level is more desirable to a buyer or service customer. Especially, the $S P C L$ value equals 1 if and only if the service quality is kept the same in a certain period. Meanwhile, the $S P C L$ value equals 0 if and only if the service quality level changes most during a certain period.

In this paper, we propose a novel service trust vector containing FTL, STT and $S P C L$ values. In addition, we also propose evaluation methods to compute the $S T T$ and $S P C L$ values.

\section{Service Trust Vector and Its Evaluation}

As discussed above, a single $F T L$ value cannot indicate the trust status of a seller or service provider well. Instead, $S T T$ and $S P C L$ values should be computed in addition to the $F T L$ value so as to provide more precise indication of the trust level to a buyer or service customer. This is the big concern of a buyer or a service customer particularly when the seller or service provider is unknown.

From another view, the service trust vector can be used to rebuild the trust data curve with a set of values, which depict the trust history well. Therefore, in addition to a single $F T L$ value, which is used in most existing trust evaluations, $S T T$ and $S P C L$ values are important as they, together with the $F T L$ value, can describe the trust data curve better.

\subsection{Final Trust Level $(F T L)$ Evaluation}

The calculation of FTL follows a common principle as follows, which appears in a number of studies [11][15].

Principle 1: The trust value is computed by taking the service quality in a recent period into account, and assigning higher weights to quality values of later services.

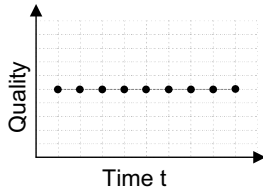

(a)

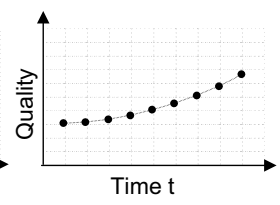

(b)

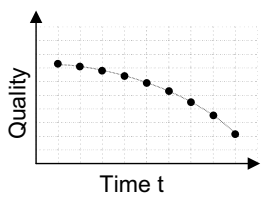

(c)

Figure 1. Several $S T T$ cases

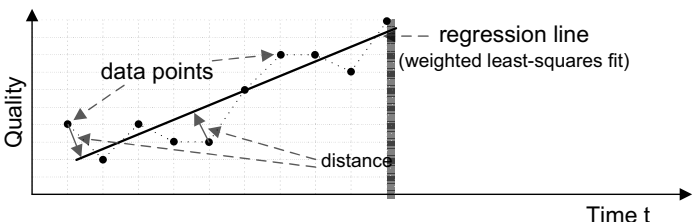

Figure 2. Weighted least squares linear regression

Based on Principle 1, the FTL value can be calculated as:

$$
T_{F T L}=\frac{\sum_{i=1}^{n} w_{i} q_{i}}{\sum_{i=1}^{n} w_{i}},
$$

where $w_{i}$ is the weight for service quality $q_{i}$ at time $i$ ( $i=$ $1 \ldots n)$, which can be calculated as follows:

$$
w_{i}=\alpha^{n-i}, \quad 0<\alpha \leq 1 .
$$

Other weighting functions can also be used, such as [12].

\subsection{Service Trust Trend ( $S T T$ ) Evaluation}

STT aims to illustrate the trust of service quality trend of changes in a given period. Some typical cases of $S T T$ are depicted in Fig. 1, which are "coherent", "up-going", "dropping" and "uncertain" in sequence.

In order to evaluate $S T T$, we refer to the idea of least squares linear regression [7]. Namely, the general trend of the data points of service quality can be represented by a regression line, which follows the general pattern of a set of data points, with no attempt to pass through each of them.

In addition, regarding Principle 1, we will introduce a weighted least squares linear regression method to evaluate $S T T$, which is illustrated in Fig. 2. This method is used to obtain the best fit straight line from a set of given data points. This best fit straight line is characterized by the sum of weighted squared residuals having its least value, where a residual is the distance from a data points to the regression line (refer to Fig. 2). Once obtaining the regression line, its slope can be taken as our $S T T$ value.

Now we introduce the $S T T$ evaluation method.

Let $\left(t_{1}, q_{1}\right),\left(t_{2}, q_{2}\right), \ldots,\left(t_{n}, q_{n}\right)$ denote the given data points in a certain period, where $q_{i}\left(q_{i} \in[0,1]\right)$ is the service quality value at time $t_{i}\left(t_{i}<t_{i+1}\right)$. Then the regression 
line can be represented as

$$
q=a_{0}+a_{1} t,
$$

where $a_{0}$ and $a_{1}$ are constants to be determined, and $a_{1}$ represents the $S T T$ value. As the distance from point $\left(t_{i}, q_{i}\right)$ to the regression line is

$$
d_{i}=\frac{\left|q_{i}-a_{0}-a_{1} t_{i}\right|}{\sqrt{1+a_{1}^{2}}}
$$

the sum of squares of the distance can be defined as follows. Definition 1: Based on the method of weighted least squares, the sum of squares of the distance can be calculated as follows:

$$
S=\sum_{i=1}^{n} w_{i}^{2} d_{i}^{2}=\sum_{i=1}^{n} \frac{w_{i}^{2}\left(q_{i}-a_{0}-a_{1} t_{i}\right)^{2}}{1+a_{1}^{2}} .
$$

Now our task is to minimize the sum of squares of the distance $S$ with respect to the parameters $a_{0}$ and $a_{1}$, with the method of undetermined coefficients.

Since function $S$ is continuous and differentiable, based on Fermat's theorem in real analysis, the minimization point of $S$ makes the first derivative of function $S$ be zero, and the second derivative positive, which could be easily proved since $a_{1}$ is very small here. For this, we differentiate $S$ with respect to $a_{0}$ and $a_{1}$, and set the results to zero, which gives

$$
\frac{\partial S}{\partial a_{0}}=-2 \sum_{i=1}^{n} \frac{w_{i}^{2}\left(q_{i}-a_{0}-a_{1} t_{i}\right)}{1+a_{1}^{2}}=0,
$$

and

$$
\frac{\partial S}{\partial a_{1}}=-2 \sum_{i=1}^{n} \frac{w_{i}^{2}\left(q_{i}-a_{0}-a_{1} t_{i}\right)\left(a_{1} q_{i}-a_{0} a_{1}+t_{i}\right)}{\left(1+a_{1}^{2}\right)^{2}}=0,
$$

i.e.

$$
\sum_{i=1}^{n} w_{i}^{2} q_{i}-a_{0} \sum_{i=1}^{n} w_{i}^{2}-a_{1} \sum_{i=1}^{n} w_{i}^{2} t_{i}=0
$$

and

$$
\begin{gathered}
\sum_{i=1}^{n} w_{i}^{2} t_{i} q_{i}+a_{0} a_{1}^{2} \sum_{i=1}^{n} w_{i}^{2} t_{i}+a_{0}^{2} a_{1} \sum_{i=1}^{n} w_{i}^{2} \\
-2 a_{0} a_{1} \sum_{i=1}^{n} w_{i}^{2} q_{i}-a_{1}^{2} \sum_{i=1}^{n} w_{i}^{2} t_{i} q_{i} \\
+a_{1} \sum_{i=1}^{n} w_{i}^{2} q_{i}^{2}-a_{0} \sum_{i=1}^{n} w_{i}^{2} t_{i}-a_{1} \sum_{i=1}^{n} w_{i}^{2} t_{i}^{2}=0 .
\end{gathered}
$$

Eqs. (6) and (7) can be solved for the unknown $a_{0}$ and $a_{1}$, by substituting $a_{0}$ from Eq. (6) into Eq. (7) to obtain

$$
a_{1}^{2}+\frac{S_{w q 2} S_{w}-S_{w q}^{2}+S_{w t}^{2}-S_{w t 2} S_{w}}{S_{w q} S_{w t}-\sum_{i=1}^{n} w_{i}^{2} t_{i} q_{i} S_{w}} a_{1}-1=0,
$$

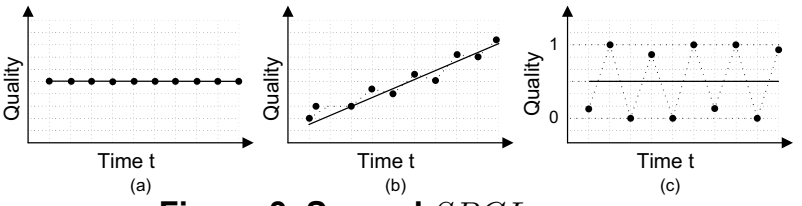

Figure 3. Several $S P C L$ cases

where $S_{w}=\sum_{i=1}^{n} w_{i}^{2}, S_{w t}=\sum_{i=1}^{n} w_{i}^{2} t_{i}, S_{w q}=$ $\sum_{i=1}^{n} w_{i}^{2} q_{i}, S_{w t 2}=\sum_{i=1}^{n} w_{i}^{2} t_{i}^{2}$, and $S_{w q 2}=\sum_{i=1}^{n} w_{i}^{2} q_{i}^{2}$. Obviously, it is easy to obtain a very small real solution of Eq. (8) about $a_{1}$. Due to the space constraint, we ignore the detailed step for calculating $a_{1}$.

Furthermore, by substituting the solution of $a_{1}$ back to Eq. (6), we can obtain

$$
a_{0}=\frac{\sum_{i=1}^{n} w_{i}^{2} q_{i}-a_{1} \sum_{i=1}^{n} w_{i}^{2} t_{i}}{\sum_{i=1}^{n} w_{i}^{2}} .
$$

Thus, based on the method of weighted least squares linear regression, we can obtain the $S T T$ value $T_{S T T}=a_{1}$, which is determined from Eq. (8). However, in order to determine the four cases of $S T T$, another factor should be taken into account. It will be introduced in Section 3.3.

\subsection{Service Performance Consistency Level $(S P C L)$ Evaluation}

The $S P C L$ value offers the indication of the consistency level of service quality in a certain period. Some typical $S P C L$ cases are depicted in Fig. 3. In sequence, they are "absolutely consistent", "relatively consistent", and "inconsistent".

Prior to presenting the detailed $S P C L$ evaluation method, we firstly introduce some definitions.

Definition 2: The predicted value of regression line is

$$
q_{t r}\left(t_{i}\right)=a_{0}+a_{1} t_{i},
$$

where $a_{0}$ and $a_{1}$ are decided by Eqs. (9) and (8) respectively.

Definition 3: According to Principle 1, the weighted average distance is

$$
q_{t d i s}=\frac{\sum_{i=1}^{n} w_{i}\left|q_{i}-q_{t r}\left(t_{i}\right)\right|}{\sqrt{1+a_{1}^{2}} \sum_{i=1}^{n} w_{i}}
$$

for $n$ quality values $q_{i}$ in period $t$.

Now let us introduce a principle about the $S P C L$ evaluation as follows.

Principle 2: The $S P C L$ value $T_{S P C L}$ is a monotonically decreasing function of the weighted mean distance $q_{t d i s}$.

According to Principle 2, we have the following SPCL evaluation formula.

Definition 4: The $S P C L$ value is

$$
T_{S P C L}=1-2 q_{t d i s}=1-2 \frac{\sum_{i=1}^{n} w_{i}\left|q_{i}-q_{t r}\left(t_{i}\right)\right|}{\sqrt{1+a_{1}^{2}} \sum_{i=1}^{n} w_{i}} .
$$


From the above definition, the properties of $S P C L$ can be analyzed as follows:

Property 1.1: If $\epsilon_{1}<T_{S P C L} \leq 1\left(0 \ll \epsilon_{1}<1\right.$ is the threshold), $S P C L$ is absolutely consistent (refer to Fig. 3(a)), i.e. the service performance remains unchanged.

Property 1.2: If $\epsilon_{2}<T_{S P C L}<\epsilon_{1}\left(0<\epsilon_{2} \ll 1\right.$ is the threshold), $S P C L$ is relatively consistent (refer to Fig. 3(b)), i.e. the service performance is consistent in a certain level.

Property 1.3: If $T_{S P C L}<\epsilon_{2}, S P C L$ is inconsistent (refer to Fig. 3(c)), i.e. the service performance is not consistent.

Now, with $T_{S T T}$ and $T_{S P C L}$, the four cases of $S T T$ can be determined as follows.

Property 2.1: If $T_{S P C L}>\epsilon_{2}$ and $\left|T_{S T T}\right|<\epsilon_{3}(0<$ $\epsilon_{3} \ll 1$ is the threshold), $S T T$ is coherent (refer to Fig. 1(a)), i.e. the service quality remains at the same level.

Property 2.2: If $T_{S P C L}>\epsilon_{2}$ and $T_{S T T}>\epsilon_{3}, S T T$ is up-going (refer to Fig. 1(b)), i.e. the service quality is becoming better.

Property 2.3: If $T_{S P C L}>\epsilon_{2}$ and $T_{S T T}<-\epsilon_{3}, S T T$ is dropping (refer to Fig. 1(c)), i.e. the service quality is turning worse.

Property 2.4: If $T_{S P C L}<\epsilon_{2}, S T T$ is uncertain (refer to Fig. 1(d)), i.e. the service quality is not reliable.

\subsection{Service Quality Trust Vector}

Based on the above discussion, we have the following definition about the service quality trust vector.

Definition 5: The service quality trust vector $\bar{T}$ consists of the $F T L$ value $T_{F T L}$, the $S T T$ value $T_{S T T}$, and the $S P C L$ value $T_{S P C L}$

$$
\bar{T}=<T_{F T L}, T_{S T T}, T_{S P C L}>,
$$

where $T_{F T L}$ is defined in Eq. (1), $T_{S T T}$ is decided by Eq. (8), and $T_{S P C L}$ is defined in Eq. (12).

Moreover, with trust vectors, all service providers form a partial order set. Given two service providers $P_{i}, P_{j}$ with service quality trust vectors $\overline{T_{i}}=<T_{F T L_{i}}, T_{S T T_{i}}$, $T_{S P C L_{i}}>$, and $\overline{T_{j}}=<T_{F T L_{j}}, T_{S T T_{j}}, T_{S P C L_{j}}>$ respectively, they are comparable in the following cases:
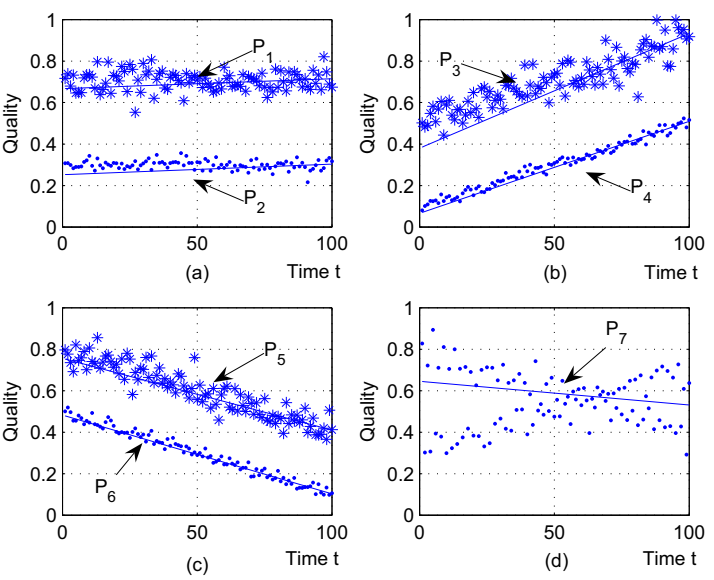

Figure 4. Study 1

1. If $T_{S T T_{i}}=T_{S T T_{j}}, T_{S P C L_{i}}=T_{S P C L_{j}}$, and $T_{F T L_{i}}<$ $T_{F T L_{j}}, P_{j}$ is more preferable. We denote it as $P_{j}>P_{i}$ or $P_{i}<P_{j}$.

2. If $T_{F T L_{i}}=T_{F T L_{j}}, T_{S P C L_{i}}=T_{S P C L_{j}}$, and $T_{S T T_{i}}<$ $T_{S T T_{j}}, P_{j}$ is more preferable. This is denoted as $P_{j}>$ $P_{i}$ or $P_{i}<P_{j}$.

3. If $T_{F T L_{i}}=T_{F T L_{j}}, T_{S T T_{i}}=T_{S T T_{j}}$, and $T_{S P C L_{i}}<$ $T_{S P C L_{j}}, P_{j}$ is more preferable. We denote it as $P_{j}>$ $P_{i}$ or $P_{i}<P_{j}$.

\section{Empirical Studies}

In this section, we illustrate the results of conducted simulations to study the proposed service trust vector approach, and explain why the service trust vector is necessary and important.

In these studies, we set $\epsilon_{1}=0.94$ and $\epsilon_{2}=0.85$ which are the thresholds to determine absolutely consistent, relatively consistent, inconsistent SPCL and uncertain STT (refer to Property 1.1-1.3\& 2.4), and set $\epsilon_{3}=0.0006$ which is the threshold for determining coherent, up-going and dropping STT together with $\epsilon_{2}$ (refer to Property 2.12.3). Meanwhile, we set the parameter $\alpha=0.95$ in the weighting function Eq. (2).

\subsection{Study 1}

In this study, we focus on four cases about $S T T$ as follows. The computed results are listed in Table 1 , and the best fit straight line for each service provider in this study is also plotted in Fig. 4.

Case 1: In this case, as plotted in Fig. 4(a), there are two service providers $P_{1}$ and $P_{2}$. According to Table 1 , as $T_{S P C L_{1}}>\epsilon_{2},\left|T_{S T T_{1}}\right|<\epsilon_{3}, T_{S P C L_{2}}>\epsilon_{2}$ 
and $\left|T_{S T T_{2}}\right|<\epsilon_{3}$, based on Property 2.1, they both have coherent $S T T$. In contrast, according to Table 1, as $\epsilon_{2}<T_{S P C L_{1}}<\epsilon_{1}$, based on Property 1.2, $P_{1}$ has relatively consistent $S P C L$. Meanwhile, as $T_{S P C L_{2}}>\epsilon_{1}$, according to Property 1.1, $P_{2}$ has $a b$ solutely consistent $S P C L$.

Case 2: As plotted in Fig. 4(b), there are two service providers $P_{3}$ and $P_{4}$ in this case. Based on Table 1, as $T_{S P C L_{3}}>\epsilon_{2}, T_{S T T_{3}}>\epsilon_{3}, T_{S P C L_{4}}>\epsilon_{2}$ and $T_{S T T_{4}}>\epsilon_{3}$, according to Property 2.2, they both have up-going STT. In contrast, based on Table 1, as $\epsilon_{2}<T_{S P C L_{3}}<\epsilon_{1}$, according to Property 1.2, $P_{3}$ has relatively consistent $S P C L$. Meanwhile, as $T_{S P C L_{4}}>\epsilon_{1}$, based on Property 1.1, $P_{4}$ has absolutely consistent $S P C L$.

Case 3: In this case, as plotted in Fig. 4(c), there are two service providers $P_{5}$ and $P_{6}$. According to Table 1 , as $T_{S P C L_{5}}>\epsilon_{2}, T_{S T T_{5}}<-\epsilon_{3}, T_{S P C L_{6}}>\epsilon_{2}$ and $T_{S T T_{6}}<-\epsilon_{3}$, based on Property 2.3, they both have dropping STT. In contrast, according to Table 1, as $\epsilon_{2}<T_{S P C L_{5}}<\epsilon_{1}$, based on Property 1.2, $P_{5}$ has relatively consistent $S P C L$. Meanwhile, as $T_{S P C L_{6}}>\epsilon_{1}$, according to Property 1.1, $P_{6}$ has $a b$ solutely consistent $S P C L$.

Case 4: As plotted in Fig. 4(d), there is one service provider $P_{7}$ in this case. According to Table 1, as $T_{S P C L_{7}}<\epsilon_{2}$, it has uncertain STT based on Property 2.4, and inconsistent SPCL based on Property 1.3 .

\begin{tabular}{|c|c|c|c|}
\hline & $T_{F T L}$ & $T_{S T T}$ & $T_{S P C L}$ \\
\hline$P_{1}$ & 0.7069 & 0.0005 & 0.9286 \\
\hline$P_{2}$ & 0.2968 & 0.0005 & 0.9573 \\
\hline$P_{3}$ & 0.8364 & 0.0055 & 0.8948 \\
\hline$P_{4}$ & 0.4288 & 0.0044 & 0.9693 \\
\hline$P_{5}$ & 0.4759 & -0.0035 & 0.9301 \\
\hline$P_{6}$ & 0.1737 & -0.0038 & 0.9710 \\
\hline$P_{7}$ & 0.5472 & -0.0011 & 0.7874 \\
\hline
\end{tabular}

Table 1. Study 1 results

\subsection{Study 2}

In this study, we introduce two cases. In Case 1, we conduct a study to illustrate why $S P C L$ is necessary and important. In Case 2, we aim to explain by examples the introduction of weight function (2) is important. The computed results are listed in Table 2 and Table 3, and the best fit straight line for each service provider in the study is also plotted in Fig. 5.

Case 1: In this case, as plotted in Fig. 5(a)(b), there are two service providers $P_{8}$ and $P_{9}$. According to Table 2, as $T_{F T L_{8}} \approx T_{F T L_{9}}, T_{S T T_{8}}=T_{S T T_{9}}$, and
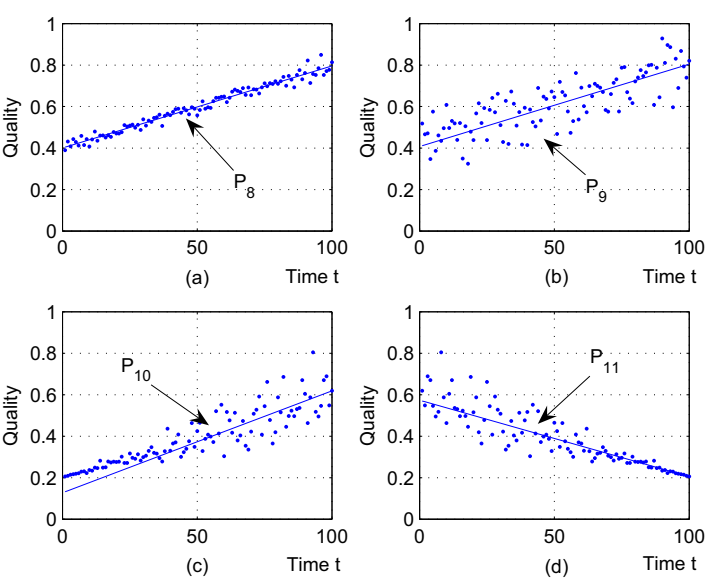

Figure 5. Study 2

\begin{tabular}{|c|c|c|c|}
\hline & $T_{F T L}$ & $T_{S T T}$ & $T_{S P C L}$ \\
\hline$P_{8}$ & 0.7232 & 0.0040 & 0.9591 \\
\hline$P_{9}$ & 0.7305 & 0.0040 & 0.8621 \\
\hline$P_{10}$ & 0.5299 & 0.0049 & 0.8661 \\
\hline$P_{11}$ & 0.2734 & -0.0037 & 0.9731 \\
\hline
\end{tabular}

Table 2. Study 2 results with $w_{i}$

$T_{S P C L_{8}}>T_{S P C L_{9}}$, we can conclude that $P_{8}>P_{9}$. This case indicates that the our $S P C L$ model is useful for depicting the trust history.

Case 2: As plotted in Fig. 5(c)(d), there are two service providers $P_{10}$ and $P_{11}$ in this case, which have the following property: the service quality $q_{i}$ at time $t_{i}$ of $P_{10}$ in Fig. 5(c) is the same as the one at time $100-t_{i}$ of $P_{11}$ in Fig. 5(d).

Without weight function (2), from Table 3, we obtain $T_{F T L_{10}}=T_{F T L_{11}}, T_{S P C L_{10}}=T_{S P C L_{11}}$ and $T_{S T T_{10}}=-T_{S T T_{11}}$. We can conclude that, without weight function (2), $P_{10}$ and $P_{11}$ have the same FTL, $S P C L$ and the absolute value of STT.

However, after introducing the weight function, we have much different values, which are listed in Table 2. As plotted in Fig. 5(c)(d), the latest $q_{i}$ of $P_{10}$ is larger than the one of $P_{11}$, which is proven by $T_{F T L_{10}}=0.5299>0.3971$ (refer to $T_{F T L_{10}}$ and $T_{F T L_{11}}$ in Table 3) $>T_{F T L_{11}}=0.2734$ in Table 2 and Table 3. Similarly, $T_{S T T_{10}}=0.0049>$ $0.0041>\left|T_{S T T_{11}}\right|=0.0037$ proves that $P_{10}$ is becoming better and $P_{11}$ is turning worse. Meanwhile, as $T_{S P C L_{10}}=0.8661<0.9126<T_{S P C L_{11}}=0.9731$, it proves that $P_{10}$ is turning less consistent and $P_{11}$ is becoming more consistent.

So we can see that with weight function (2) the service quality history can be described more precisely. 


\begin{tabular}{|c|c|c|c|}
\hline & $T_{F T L}$ & $T_{S T T}$ & $T_{S P C L}$ \\
\hline$P_{8}$ & 0.6003 & 0.0040 & 0.9687 \\
\hline$P_{9}$ & 0.6133 & 0.0035 & 0.8635 \\
\hline$P_{10}$ & 0.3971 & 0.0041 & 0.9126 \\
\hline$P_{11}$ & 0.3971 & -0.0041 & 0.9126 \\
\hline
\end{tabular}

Table 3. Study 2 results without $w_{i}$
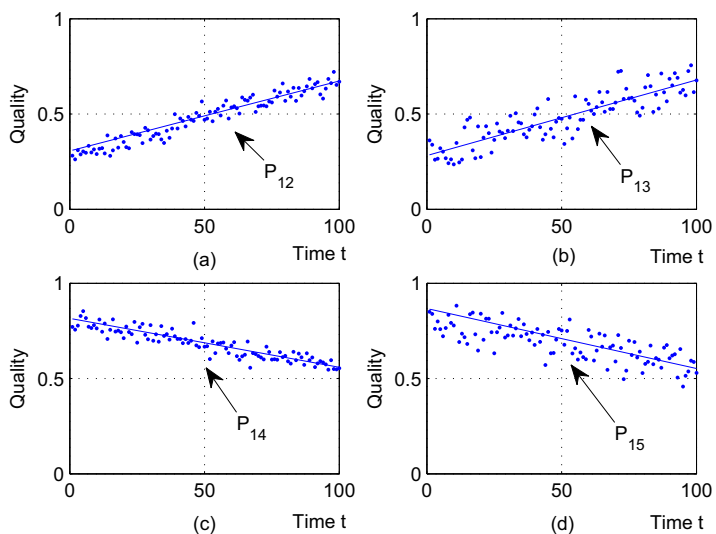

(c)

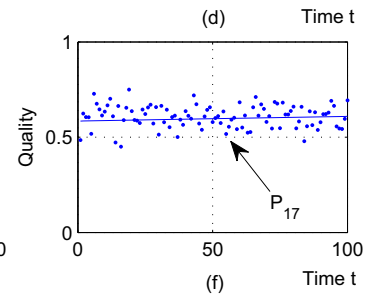

Figure 6. Study 3

\subsection{Study 3}

In this study, we conduct a study with six cases to illustrate why the trust vector is necessary and important. The computed results are listed in Table 4, and the best fit straight line for each service provider in this study is also plotted in Fig. 6.

\begin{tabular}{|c|c|c|c|}
\hline & $T_{F T L}$ & $T_{S T T}$ & $T_{S P C L}$ \\
\hline$P_{12}$ & 0.6055 & 0.0037 & 0.9472 \\
\hline$P_{13}$ & 0.6059 & 0.0040 & 0.8966 \\
\hline$P_{14}$ & 0.6053 & -0.0026 & 0.9602 \\
\hline$P_{15}$ & 0.6076 & -0.0032 & 0.9015 \\
\hline$P_{16}$ & 0.6092 & -0.0001 & 0.9417 \\
\hline$P_{17}$ & 0.6065 & 0.0003 & 0.9039 \\
\hline
\end{tabular}

Table 4. Study 3 results

In each case, as plotted in Fig. 6, there is one service provider. According to Table 4, all six cases have almost the same FTL, but different $T_{S T T}$ or $T_{S P C L}$. Based on Properties 1.1-1.3 \& 2.1-2.3, we can determine the typical cases of $S T T$ and $S P C L$ in Table 5. Meanwhile, we can conclude that $P_{12}>P_{13}, P_{12}>P_{14}, P_{13}>P_{15}, P_{14}>$ $P_{15}, P_{16}>P_{14}, P_{16}>P_{17}$, and $P_{17}>P_{15}$. Namely, with
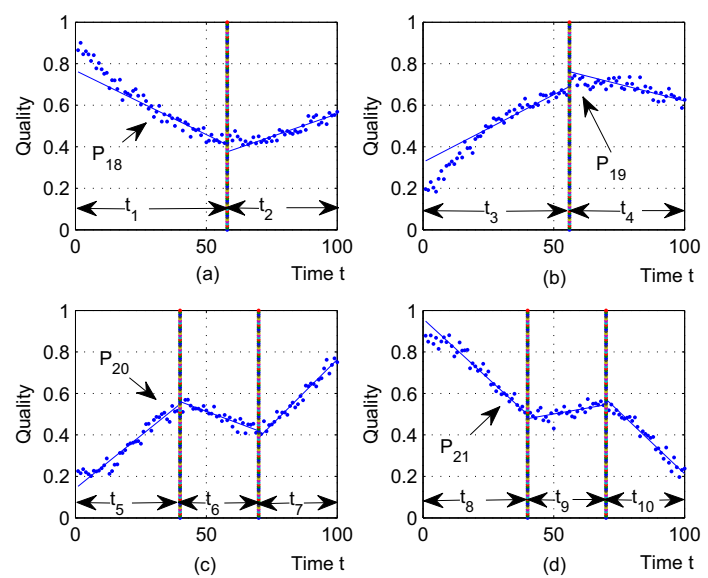

Figure 7. Study 4

solo $F T L$, it is not likely to depict the trust history exactly.

\begin{tabular}{|c|c|c|}
\hline & $T_{S T T}$ & $T_{S P C L}$ \\
\hline$P_{12}$ & up-going & absolutely consistent \\
\hline$P_{13}$ & up-going & relatively consistent \\
\hline$P_{14}$ & dropping & absolutely consistent \\
\hline$P_{15}$ & dropping & relatively consistent \\
\hline$P_{16}$ & coherent & absolutely consistent \\
\hline$P_{17}$ & coherent & relatively consistent \\
\hline
\end{tabular}

Table 5. Study 3 results about typical cases

Therefore, in this study, we can notice that the trust vector including $T_{F T L}, T_{S T T}$ and $T_{S P C L}$ can describe the history of trust data more precisely, than the solo FTL.

\subsection{Study 4}

Until now we consider only one time period $t$ in all cases. In order to evaluate the trust history of service quality better, multiple time periods may have to be introduced, where each period corresponds to the trust trend with high $T_{S P C L}$ value. There are four cases in this study. The computed results are listed in Table 6 . The best fit straight line for each case with one service provider is also plotted in Fig. 7.

According to Table 6, based on Properties 2.1-2.3, we can determine the typical cases of STT in Table 7. Obviously, it is easy to see that each period has absolutely consistent $S P C L$ (i.e. high $T_{S P C L}$ value).

From the above results, we can see that in case of dynamic trust trend, proper multiple time periods should be determined so as to describe the transaction history better.

\section{Conclusions}

In this paper, we propose a trust vector approach to service-oriented applications, which includes final trust level (FTL), service trust trend (STT), and service performance consistency level (SPCL). Corresponding evalua- 


\begin{tabular}{|c|c|c|c|c|}
\hline \multicolumn{2}{|l|}{} & $T_{F T L}$ & $T_{S T T}$ & $T_{S P C L}$ \\
\hline$P_{18}$ & $t_{1}$ & 0.5107 & -0.0062 & 0.9590 \\
\hline & $t_{2}$ & 0.4998 & 0.0044 & 0.9663 \\
\hline$P_{19}$ & $t_{3}$ & 0.5842 & 0.0065 & 0.9534 \\
\hline & $t_{4}$ & 0.6646 & -0.0032 & 0.9615 \\
\hline$P_{20}$ & $t_{5}$ & 0.4159 & 0.0102 & 0.9545 \\
\hline & $t_{6}$ & 0.4717 & -0.0047 & 0.9683 \\
\hline & $t_{7}$ & 0.6246 & 0.0126 & 0.9655 \\
\hline$P_{21}$ & $t_{8}$ & 0.6477 & -0.0116 & 0.9622 \\
\hline & $t_{9}$ & 0.5206 & 0.0022 & 0.9649 \\
\hline & $t_{10}$ & 0.3449 & -0.0120 & 0.9567 \\
\hline
\end{tabular}

Table 6. Study 4 results

\begin{tabular}{|l|l|c|}
\hline \multicolumn{2}{|l|}{} & $T_{S T T}$ \\
\hline$P_{18}$ & $t_{1}=[0,58]$ & dropping \\
\hline & $t_{2}=[58,100]$ & up-going \\
\hline$P_{19}$ & $t_{3}=[0,56]$ & up-going \\
\hline & $t_{4}=[56,100]$ & dropping \\
\hline$P_{20}$ & $t_{5}=[0,40]$ & up-going \\
\hline & $t_{6}=[40,70]$ & dropping \\
\hline & $t_{7}=[70,100]$ & up-going \\
\hline$P_{21}$ & $t_{8}=[0,40]$ & dropping \\
\hline & $t_{9}=[40,70]$ & up-going \\
\hline & $t_{10}=[70,100]$ & dropping \\
\hline
\end{tabular}

Table 7. Study 4 results about typical $S T T$

tion methods for $S T T$ and $S P C L$ are also proposed. From our analytical and empirical studies, we can see that the proposed approach can depict trust history exactly. It offers more information to service customers for their decisionmaking in the selection of trustworthy service providers.

For future work, as we have discussed in Section 4.4, a multiple time period detection algorithm should be studied for the case with various trust trends, with which an accurate trust vector can be determined for each period.

\section{References}

[1] eBay. http://www.eBay.com/.

[2] GNutella. http://www.gnutella.com/.

[3] E. Damiani, S. D. C. di Vimercati, S. Paraboschi, P. Samarati, and F. Violante. A reputation based approach for choosing reliable resources in peertopeer networks. In Proceedings of $A C M C C S^{\prime} 02$, pages 207-216, Washington DC, USA, November 2002.

[4] S. D. Kamvar, M. T. Schlosser, and H. Garcia-Molina. The eigentrust algorithm for reputation management in P2P networks. In Proceedings of the 12th International $W W W$ Conference, Budapest, Hungary, May 2003.
[5] K.-J. Lin, H. Lu, T. Yu, and C. en Tai. A reputation and trust management broker framework for web applications. In Proceedings of The 2005 IEEE International Conference on e-Technology, e-Commerce and e-Service (EEE'05), pages 262-269, March 2005.

[6] S. Marti and H. Garcia-Molina. Limited reputation sharing in P2P systems. In Proceedings of $A C M$ $E C^{\prime} 04$, pages 91-101, New York, USA, May 2004.

[7] S. Rao. Applied Numerical Methods for Engineers and Scientists. Prentice Hall, 2002.

[8] S. Song, K. Hwang, R. Zhou, and Y.-K. Kwok. Trusted $\mathrm{p} 2 \mathrm{p}$ transactions with fuzzy reputation aggregation. IEEE Internet Computing, 9(6):24-34, 2005.

[9] L.-H. Vu, M. Hauswirth, and K. Aberer. Qos-based service selection and ranking with trust and reputation management. In Proceedings of 13th International Conference on Cooperative Information Systems (CoopIS 2005), Oct 31-Nov 42005.

[10] Y. Wang, K.-J. Lin, D. S. Wong, and V. Varadharajan. The design of a rule-based and event-driven trust management framework. In The IEEE International Conference on e-Business Engineering (ICEBE 2007), pages 97-104, Hong Kong, October 2007.

[11] Y. Wang and V. Varadharajan. Interaction trust evaluation in decentralized environments. In K. Bauknecht, M. Bichler, and B. Pröll, editors, Proceedings of 5th International Conference on Electronic Commerce and Web Technologies (EC-Web'04), volume LNCS 3182, Springer-Verlag, pages 144-153, Zaragoza, Spain, August-September 2004.

[12] Y. Wang and V. Varadharajan. Trust ${ }^{2}$ : Developing trust in peer-to-peer environments. In Proceedings of 2005 IEEE International Conference on Services Computing (SCC 2005), pages 24-31, Orlando, Florida, USA, July 2005.

[13] L. Xiong and L. Liu. PeerTrust: Supporting reputation-based trust for peer-to-peer electronic communities. IEEE Trans. on Knowlege and Data Engineering, 16(7):843-857, 2004.

[14] B. Yu, M. P. Singh, and K. Sycara. Developing trust in large-scale peer-to-peer systems. In Proceedings of 2004 IEEE First Symposium on Multi-Agent Security and Survivability, pages 1-10, August 2004.

[15] G. Zacharia and P. Maes. Trust management through reputation mechanisms. Applied Artificial Intelligence Journal, 9:881-908, 2000. 